\title{
ON LEXICOGRAPHICALLY SHELLABLE POSETS
}

BY

\author{
ANDERS BJORNER AND MICHELLE WACHS ${ }^{1}$
}

\begin{abstract}
Lexicographically shellable partially ordered sets are studied. A new recursive formulation of CL-shellability is introduced and exploited. It is shown that face lattices of convex polytopes, totally semimodular posets, posets of injective and normal words and lattices of bilinear forms are CL-shellable. Finally, it is shown that several common operations on graded posets preserve shellability and CL-shellability.
\end{abstract}

1. Introduction. A finite poset (partially ordered set) $P$ is said to be shellable if all maximal chains have the same length $r$ and can be ordered $m_{1}, m_{2}, \ldots, m_{t}$ in such a way that if $1 \leqslant i<j \leqslant t$ then there exist $1 \leqslant k<j$ and $x \in m_{j}$ such that $m_{i} \cap m_{j} \subseteq$ $m_{k} \cap m_{j}=m_{j}-\{x\}$. A shellable poset enjoys several strong properties of a combinatorial, topological and algebraic nature. Let it suffice here to mention that the order complex $\Delta(P)$ has the homotopy type of a wedge of $r$-spheres and that a naturally associated commutative ring is Cohen-Macaulay if $P$ is shellable. The papers [1, 2 and 8] and the further references mentioned there provide more details.

In [1] a simple method was described for showing that a graded poset $P$ is shellable. The method consists in labeling the covering relations of $P$ in a certain favorable way. When this is possible $P$ is said to be EL-shellable (or, "edge lexicographically shellable", cf. Definition 2.1). A slightly more general version of the method was formulated in [2] leading to the concept $C L$-shellable (or, "chain lexicographically shellable", cf. Definition 2.2). In this paper we continue the study of lexicographic shellability, the main result being a new recursive formulation of $C L$-shellability (\$3). Using this tool we are able to prove that face lattices of convex polytopes (\$4), totally semimodular posets (\$5), posets of injective words (\$6) and lattices of bilinear forms (\$7) are CL-shellable.

In [1] the question was raised, whether face lattices of convex polytopes are lexicographically shellable? It is shown in $\$ 4$ that a polyhedral complex is shellable (in a certain recursive sense, which is essentially that of Bruggesser and Mani [3]) if and only if the dual of its face lattice is CL-shellable. Hence, somewhat surprisingly, asking for the CL-shellability of a polytope's face lattice turns out to be equivalent to asking for the shellability of the dual polytope's boundary complex.

In a final section (\$8) we prove that a number of common poset operations on graded posets preserve shellability and CL-shellability. The results complement

Received by the editors April 29, 1982.

1980 Mathematics Subject Classification. Primary 05A99, 06A10; Secondary 52A25, 57Q05, 68F99.

' Partially supported by NSF MCS 81-03474. 
those of $[1, \S 4]$. For instance, it is shown that rank-selection preserves CL-shellability. This fact, together with [2], implies that rank-selected infinite Bruhat orders and posets of normal words are CL-shellable (\$6).

2. Preliminaries. Let $P$ be a finite poset. We say that $P$ is bounded if there exist a top element $\hat{1} \in P$ and a bottom element $\hat{0} \in P$ such that $\hat{0} \leqslant x \leqslant \hat{1}$ for all $x \in P$. Given any poset $P$, let $\hat{P}$ denote the bounded poset obtained from $P$ by adjoining a bottom element $\hat{0}$ and a top element $\hat{1} . P$ is said to be pure if all maximal chains $x_{0}<x_{1}<\cdots<x_{r}$ have the same length $r$. A finite poset $P$ is said to be graded if it is bounded and pure. Any element $x$ of a graded poset $P$ has a well-defined rank $\rho(x)$ equal to the common length of all unrefinable chains from $\hat{0}$ to $x$. By the length of $P$ we mean $\rho(\hat{1})$. We say that $y$ covers $x$ in $P$ and write $x \rightarrow y$ if $x<y$ and $x<z \leqslant y$ implies that $z=y$. The set of all chains of a poset $P$ will be denoted by $\mathcal{C}(P)$ and the set of all maximal chains by $\Re(P)$. The dual of a poset $P$ will be denoted by $P^{*}$.

Let $\Delta$ be a finite simplicial complex. The maximal faces of $\Delta$ are called facets. We say that $\Delta$ is pure d-dimensional if all facets are of dimension $d$; that is, they contain $d+1$ vertices. A pure $d$-dimensional simplicial complex $\Delta$ is said to be shellable if its facets can be ordered $F_{1}, F_{2}, \ldots, F_{t}$ in such a way that $\bar{F}_{j} \cap \cup_{i=1}^{j-1} \bar{F}_{i}$ is a pure $(d-1)$-dimensional complex for $j=2,3, \ldots, t\left(\bar{F}_{j}\right.$ denotes the set $\left.\left\{G \mid G \subseteq F_{j}\right\}\right)$. Such an ordering of facets is called a shelling. The following equivalent formulation of shellability will be used throughout this paper: A linear ordering $\Omega$ of the facets of a pure simplicial complex is a shelling if and only if $\Omega$ satisfies the following property.

Property S. For all facets $F$ and $F^{\prime}$ of $\Delta$ such that $F^{\prime}<\Omega F$ there is a facet $F^{\prime \prime}$ with $F^{\prime \prime}<^{\Omega} F$ such that $F^{\prime} \cap F \subseteq F^{\prime \prime} \cap F$ and $\left|F^{\prime \prime} \cap F\right|=|F|-1$.

To a finite poset $P$ one can associate the simplicial complex $\Delta(P)$ of all chains of $P$, called the order complex of $P$. Clearly, the facets of $\Delta(P)$ are the maximal chains of $P$. Also, if $P$ is a graded poset of length $n$ then $\Delta(P)$ is pure $n$-dimensional. We say that a finite pure poset $P$ is shellable if its order complex $\Delta(P)$ is shellable. Note that a finite poset $P$ is shellable if and only if $\hat{P}$ is shellable.

The cardinality of a finite set $A$ will be denoted by $|A|$. For a positive integer $n$, let $[n]=\{1,2, \ldots, n\}$.

Let us now review the notion of lexicographic shellability, starting with the simpler and original version defined in [1]. Let $P$ be a graded poset of length $n$, and let $\mathcal{E}(P)$ be the set of edges of the Hasse diagram of $P$, i.e., $\mathcal{E}(P)=\{(x, y) \in P \times$ $P \mid x \rightarrow y\}$. An edge labeling of $P$ is a map $\lambda: \mathcal{E}(P) \rightarrow \Lambda$ where $\Lambda$ is some poset (usually the integers). Given an edge labeling $\lambda$, each unrefinable chain $c=\left(x_{0} \rightarrow x_{1}\right.$ $\left.\rightarrow \cdots \rightarrow x_{k}\right)$ of length $k$ can be associated with a $k$-tuple $\sigma(c)=\left(\lambda\left(x_{0}, x_{1}\right)\right.$, $\left.\lambda\left(x_{1}, x_{2}\right), \ldots, \lambda\left(x_{k-1}, x_{k}\right)\right)$. We say that $c$ is an increasing chain if the $k$-tuple $\sigma(c)$ is increasing; that is, if $\lambda\left(x_{0}, x_{1}\right) \leqslant \lambda\left(x_{1}, x_{2}\right) \leqslant \cdots \leqslant \lambda\left(x_{k-1}, x_{k}\right)$. The edge labeling allows us to order the maximal chains of any interval of $P$ by ordering the corresponding $k$-tuples lexicographically. If $\sigma\left(c_{1}\right)$ lexicographically precedes $\sigma\left(c_{2}\right)$ then we say that $c_{1}$ lexicographically precedes $c_{2}$ and we denote this by $c_{1}<_{L} c_{2}$. 
Definition 2.1. An edge labeling is called an EL-labeling (edge lexicographical labeling) if for every interval $[x, y]$ in $P$,

(i) there is a unique increasing maximal chain $c$ in $[x, y]$, and

(ii) $c<_{L} c^{\prime}$ for all other maximal chains $c^{\prime}$ in $[x, y]$.

A graded poset that admits an EL-labeling is said to be EL-shellable (edge lexicographically shellable).

An example of an integer EL-labeling of the face lattice of a square is given in Figure 2.1.

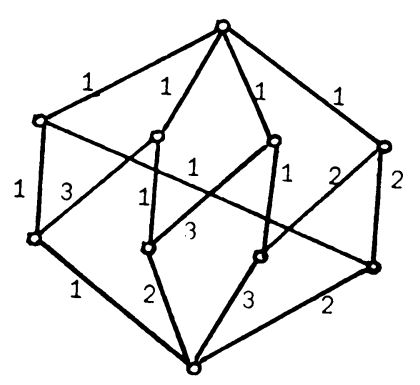

FIGURE 2.1

For a graded poset $P$ of length $n$ let $\mathcal{E}^{*}(P)$ be the set of edges of maximal chains of $P$, i.e., $\mathcal{E}^{*}(P)=\{(c, x, y) \mid c \in \Re(P), x, y \in c, x \rightarrow y\}$. A chain-edge labeling of $P$ is a map $\lambda: \mathcal{E}^{*}(P) \rightarrow \Lambda$, where $\Lambda$ is some poset (usually the integers), that satisfies the following condition.

Condition L. If two maximal chains $c=\left(\hat{0}=x_{0} \rightarrow x_{1} \rightarrow \cdots \rightarrow x_{n}=\hat{1}\right)$ and $c^{\prime}=$ $\left(\hat{0}=x_{0}^{\prime} \rightarrow x_{1}^{\prime} \rightarrow \cdots \rightarrow x_{n}^{\prime}=\hat{1}\right)$ coincide along their first $d$ edges then their labels also coincide along these edges; that is, if $x_{i}=x_{i}^{\prime}$ for $i=0, \ldots, d$ then $\lambda\left(c, x_{i-1}, x_{i}\right)$ $=\lambda\left(c^{\prime}, x_{i-1}^{\prime}, x_{i}^{\prime}\right)$ for $i=1, \ldots, d$.

An example of a chain-edge labeling is given in Figure 2.2.
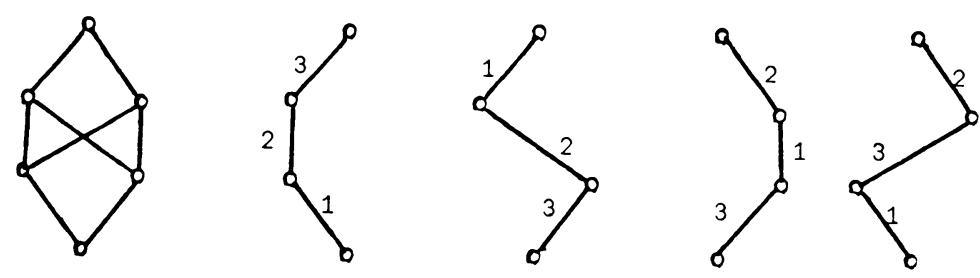

FIGURE 2.2

Let $\lambda$ be a chain-edge labeling of $P$. Each maximal chain $m=\left(\hat{0}=x_{0} \rightarrow x_{1} \rightarrow\right.$ $\left.\cdots \rightarrow x_{n}=\hat{1}\right)$ of $P$ can be associated with a unique $n$-tuple

$$
\sigma(m)=\left(\lambda\left(m, x_{0}, x_{1}\right), \lambda\left(m, x_{1}, x_{2}\right), \ldots, \lambda\left(m, x_{n-1}, x_{n}\right)\right) .
$$

Unrefinable chains of length $k<n$ cannot however be directly associated with unique $k$-tuples as in the edge labeling case. If $c$ is an unrefinable chain of length $k<n$, then each maximal chain containing $c$ induces a $k$-tuple to be associated with $c$. We overcome the problem of uniqueness by extending the concept of interval. If 
$[x, y]$ is an interval and $r$ is an unrefinable chain from $\hat{0}$ to $x$, then the pair $([x, y], r)$ will be called a rooted interval with root $r$, and will be denoted $[x, y]_{r}$. If $c$ is any maximal chain of $[x, y]$ then $r \cup c$ is a maximal chain of $[\hat{0}, y]$. By Condition $\mathrm{L}$ if $m$ and $m^{\prime}$ are maximal chains that contain $r \cup c$, then the first $d$ entries of $\sigma(m)$ and $\sigma\left(m^{\prime}\right)$ coincide, where $d$ is the length of the chain $r \cup c$. Hence all maximal chains that contain $r$ and $c$ induce the same $k$-tuple to be associated with $c$. This implies that every maximal chain $c$ in a rooted interval $[x, y]_{r}$ has a unique $k$-tuple $\sigma_{r}(c)$ associated with it.

We say that a maximal chain $c$ in a rooted interval $[x, y]_{r}$ is increasing if the $k$-tuple $\sigma_{r}(c)$ is increasing. If $c_{1}$ and $c_{2}$ are maximal chains of $[x, y]_{r}$ then $c_{1}$ is said to lexicographically precede $c_{2}$ in $[x, y]_{r}$ if $\sigma_{r}\left(c_{1}\right)$ lexicographically precedes $\sigma_{r}\left(c_{2}\right)$. We denote this by $c_{1}<_{L} c_{2}$ in $[x, y]_{r}$.

Definition 2.2. A chain-edge labeling $\lambda$ is called a $C L$-labeling (chain lexicographical labeling) if for every rooted interval $[x, y]_{r}$ in $P$,

(i) there is a unique increasing maximal chain $c$ in $[x, y]_{r}$, and

(ii) $c<_{L} c^{\prime}$ for all other maximal chains $c^{\prime}$ in $[x, y]_{r}$.

A graded poset is said to be $C L$-shellable (chain lexicographically shellable) if it admits a CL-labeling.

Figure 2.2 shows an example of a CL-labeling. The notion of CL-shellability was first used in [2]. For emphasis we will state the logical relationship between the three notions of shellability for a graded poset. The first implication is obvious, the second is proved in [2, Theorem 3.3]. See $\$ 9$ for some additional remarks.

Proposition 2.3. EL-shellable $\Rightarrow C L$-shellable $\Rightarrow$ shellable.

Some examples of EL-shellable posets are semimodular lattices (including all modular and geometric lattices) and supersolvable lattices (see [1]). A class of CL-shellable posets that are not in general known to be EL-shellable are the duals of Bruhat order on finite Coxeter groups (see [2]).

We will call a poset $P$ dual $E L$-shellable [CL-shellable] if its dual poset $P^{*}$ is EL-shellable [CL-shellable]. Thus the Bruhat order on a finite Coxeter group is dual CL-shellable.

In the next section we will see that the concept of CL-shellability is not made more general by allowing $\Lambda$ to be an arbitrary poset rather than the set of integers. It is however useful in proving some results to allow $\Lambda$ to be an arbitrary poset. It is not known to us whether the concept of EL-shellability would be affected by restricting the poset $\Lambda$ to the integers.

3. Recursive atom orderings. The fact that every interval of a CL-shellable poset is CL-shellable leads to the question of whether CL-shellability can be formulated recursively. It turns out that the following recursive property is equivalent to CL-shellability. Recall that the atoms of a graded poset are the elements which cover $\hat{0}$. Dually, the coatoms are the elements which are covered by $\hat{1}$.

Definition 3.1. A graded poset $P$ is said to admit a recursive atom ordering if the length of $P$ is 1 or if the length of $P$ is greater than 1 and there is an ordering $a_{1}, a_{2}, \ldots, a_{t}$ of the atoms of $P$ which satisfies: 
(i) For all $j=1,2, \ldots, t,\left[a_{j}, \hat{1}\right]$ admits a recursive atom ordering in which the atoms of $\left[a_{j}, \hat{1}\right]$ that come first in the ordering are those that cover some $a_{i}$ where $i<j$.

(ii) For all $i<j$, if $a_{i}, a_{j}<y$ then there is a $k<j$ and an element $z$ such that $a_{k}$, $a_{j} \rightarrow z \leqslant y$.

If $a_{1}, a_{2}, \ldots, a_{t}$ is an ordering of the atoms of $P$ that satisfies (i) and (ii) then $a_{1}, a_{2}, \ldots, a_{t}$ is said to be a recursive atom ordering.

An example of a poset which admits a recursive atom ordering is given in Figure 3.1(a). It is easy to see that (i) and (ii) of Definition 3.1 are satisfied if the atoms are ordered from left to right in the Hasse diagram. Figure 3.1(b) gives an example of a poset that does not admit a recursive atom ordering, since for any ordering of the atoms, (ii) cannot be satisfied.

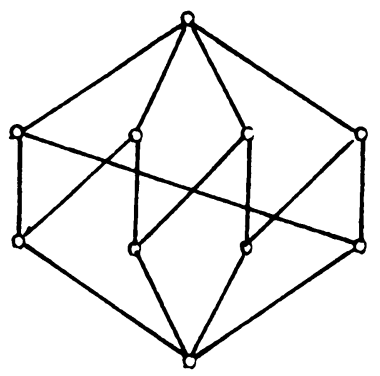

(a)

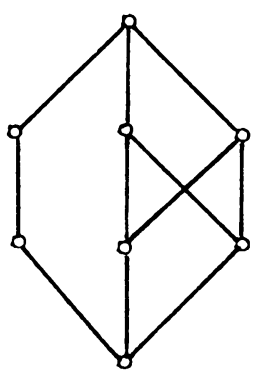

(b)

Figure 3.1

We will also be considering recursive coatom orderings. A poset admits a recursive coatom ordering if its dual admits a recursive atom ordering.

TheOREM 3.2. A graded poset $P$ admits a recursive atom ordering if and only if $P$ is CL-shellable.

Proof. We begin with the "only if" part. We will prove the following statement by induction on the length of $P$ : Any integer labeling $\lambda$ of the bottom edges of a graded poset $P$ which admits a recursive atom ordering $a_{1}, a_{2}, \ldots, a_{t}$ extends to an integer CL-labeling of $P$ if $\lambda\left(\hat{0}, a_{i}\right)<\lambda\left(\hat{0}, a_{j}\right)$ for all $i<j$. This clearly holds for posets of length 1 .

Now assume that $P$ has length greater than 1 . For each $j$, let $F\left(a_{j}\right)$ be the set of all atoms of $\left[a_{j}, \hat{1}\right]$ that cover some $a_{i}$ where $i<j$. By (i) of Definition 3.1, the atoms of $F\left(a_{j}\right)$ come first in some recursive atom ordering of $\left[a_{j}, \hat{1}\right]$. We can thus label the bottom edges of $\left[a_{j}, \hat{1}\right]$ consistently with the atom ordering of $\left[a_{j}, \hat{1}\right]$ and satisfying,

$$
\begin{aligned}
& x \in F\left(a_{j}\right) \Rightarrow \lambda\left(a_{j}, x\right)<\lambda\left(\hat{0}, a_{j}\right), \\
& x \notin F\left(a_{j}\right) \Rightarrow \lambda\left(a_{j}, x\right)>\lambda\left(\hat{0}, a_{j}\right),
\end{aligned}
$$

where $\lambda$ denotes the labeling of the bottom edges of $\left[a_{j}, \hat{1}\right]$ as well as the original labeling of the bottom edges of $P$. 
By the induction hypothesis this labeling extends to an integer CL-labeling of $\left[a_{j}, \hat{1}\right]$. Choosing such an extension at each $a_{j}$ we obtain a chain-edge labeling $\lambda$ of $P$ which is a CL-labeling of $\left[a_{j}, \hat{1}\right]$ for all $j=1, \ldots, t$, and hence for every rooted interval whose bottom element is not $\hat{0}$, and which extends the original labeling of the bottom edges of $P$.

We need only show now that the unique lexicographically first maximal chain in any interval $[\hat{0}, y]$ is the only increasing maximal chain in that interval. Let $c=\left(\hat{0} \rightarrow x_{1} \rightarrow x_{2} \rightarrow \cdots \rightarrow x_{k}=y\right)$ be the lexicographically first maximal chain in $[\hat{0}, y]$. Then $\left(x_{1} \rightarrow x_{2} \rightarrow \cdots \rightarrow x_{k}\right)$ is the lexicographically first maximal chain in $\left[x_{1}, y\right]$ and is therefore increasing. It is also true that $x_{2} \notin F\left(x_{1}\right)$ since $c$ is lexicographically first. Thus, by (3.3), $\lambda\left(\hat{0}, x_{1}\right)<\lambda\left(x_{1}, x_{2}\right)$ and hence $c$ is increasing.

If $c^{\prime}=\left(\hat{0} \rightarrow x_{1}^{\prime} \rightarrow x_{2}^{\prime} \rightarrow \cdots \rightarrow x_{k}^{\prime}=y\right)$ is another increasing maximal chain in $[\hat{0}, y]$ then $x_{1}^{\prime} \neq x_{1}$ because there is only one increasing maximal chain in $\left[x_{1}, y\right]$. It also follows that $x_{1}^{\prime} \rightarrow x_{2}^{\prime} \rightarrow \cdots \rightarrow x_{k}^{\prime}$ is the lexicographically first maximal chain in $\left[x_{1}^{\prime}, y\right]$. Consequently, $x_{2}^{\prime}$ is the first atom in the recursive atom ordering of $\left[x_{1}^{\prime}, \hat{1}\right]$ that is less than $y$. Since $0 \rightarrow x_{1}^{\prime} \rightarrow x_{2}^{\prime}$ is increasing $x_{2}^{\prime} \notin F\left(x_{1}^{\prime}\right)$ by (3.3), and hence $F\left(x_{1}^{\prime}\right)$ has no elements which lie below $y$. This contradicts (ii) of Definition 3.1, since $x_{1}$ precedes $x_{1}^{\prime}$ in the given recursive atom ordering of $P$. Therefore $c$ is the only increasing maximal chain in $[\hat{0}, y]$, and hence $P$ is CL-shellable.

To prove the converse we let $\lambda: \mathcal{E}^{*}(P) \rightarrow \Lambda$ be a CL-labeling of $P$, where $\Lambda$ is an arbitrary poset. If $\lambda$ is applied to the bottom edges of $P$, a partial ordering of the atoms of $P$ is induced. We say that an atom ordering $a_{1}, a_{2}, \ldots, a_{t}$ is compatible with the CL-labeling $\lambda$ if $a_{1}, a_{2}, \ldots, a_{t}$ is a linear extension of the partial ordering induced by $\lambda$, i.e., $a_{1}, a_{2}, \ldots, a_{t}$ is compatible with $\lambda$ if $\lambda\left(\hat{0}, a_{i}\right)<\lambda\left(\hat{0}, a_{j}\right)$ implies that $i<j$.

We prove that if the atom ordering $a_{1}, a_{2}, \ldots, a_{t}$ is compatible with $\lambda$ then $a_{1}, a_{2}, \ldots, a_{t}$ is a recursive atom ordering. The proof is by induction on the length of $P$. The statement holds trivially if the length of $P$ is 1 . Let $P$ have length greater than 1. For each $j=1,2, \ldots, t,\left[a_{j}, \hat{1}\right]$ is CL-shellable with CL-labeling $\lambda$ inherited from the CL-labeling of $P$. Hence by induction any atom ordering of $\left[a_{j}, \hat{1}\right]$ that is compatible with $\lambda$ is a recursive atom ordering. We must now find an atom ordering of $\left[a_{j}, \hat{1}\right]$ that is both compatible with $\lambda$ and satisfies Definition 3.1(i).

If $x \in F\left(a_{j}\right)$ then $\hat{0} \rightarrow a_{j} \rightarrow x$ is not the lexicographically first maximal chain in $[\hat{0}, x]$, since $a_{1}, a_{2}, \ldots, a_{t}$ is compatible with $\lambda$. Hence, $\hat{0} \rightarrow a_{j} \rightarrow x$ is not increasing and $\lambda\left(\hat{0}, a_{j}\right) \neq \lambda\left(a_{j}, x\right)$. If $y \notin F\left(a_{j}\right)$ then $\hat{0} \rightarrow a_{j} \rightarrow y$ is the lexicographically first maximal chain in $[\hat{0}, y]$ and hence is increasing. Thus $\lambda\left(\hat{0}, a_{j}\right) \leqslant \lambda\left(a_{j}, y\right)$. The two inequalities combine to $\lambda\left(a_{j}, x\right) \neq \lambda\left(a_{j}, y\right)$. Therefore the atoms of $\left[a_{j}, \hat{1}\right]$ can be ordered compatibly with $\lambda$ and satisfying (i) of Definition 3.1. By induction this ordering is recursive.

To verify Definition 3.1(ii) we take $i<j$ and $a_{i}, a_{j}<y$ and let $c$ be the lexicographically first maximal chain in $\left[a_{j}, y\right]$. Since the atoms $a_{1}, a_{2}, \ldots, a_{t}$ are ordered compatibly with $\lambda, \hat{0} \cup c$ cannot be the lexicographically first maximal chain in $[\hat{0}, y]$, and hence $\hat{0} \cup c$ has a descent which can occur only at $a_{j}$. Let $z$ be the element on $c$ that covers $a_{j}$. Since there is a descent at $a_{j}, z$ covers some $a_{k}$ where $k<j$, and (ii) of Definition 3.1 follows. 
In the last two paragraphs we have tacitly used the fact that if $a_{i}, a_{j}<z, a_{i} \neq a_{j}$, and the lexicographically first maximal chain in $[\hat{0}, z]$ contains $a_{i}$, then $\lambda\left(\hat{0}, a_{i}\right)<$ $\lambda\left(\hat{0}, a_{j}\right)$. This simple property of CL-shellability can be verified as in [1, Proposition 2.5].

A consequence of the preceding proof is that the labeling poset $\Lambda$ for a CL-shellable poset can always without loss of generality be taken to be the totally ordered set of integers. This is because in the first part of the proof we produced an integer labeling.

4. Face lattices of complexes. The recursive formulation of CL-shellability is well suited to deal with posets which seem to lack natural (chain-) edge labelings but do exhibit good recursive properties. The face lattices of simplicial and polyhedral complexes illustrate this point. Other examples will be given in later sections.

By a (convex) polytope is meant the convex hull of a finite set of points in Euclidean space. A polyhedral complex is defined to be a finite set of polytopes in some Euclidean space such that a face of a member is a member (including the empty face) and the intersection of any two members is a face of each. The maximal faces of a polyhedral complex are called facets. If the dimension of all the facets of a polyhedral complex $\Delta$ is $d$ then $\Delta$ will be called simply a $d$-complex. Note that if all the facets of a $d$-complex are simplices then we have (a geometric realization of) a usual simplicial complex. The $d$-complex consisting of a $d$-dimensional polytope $P$ and all its faces will be denoted by $\bar{P}$. The boundary complex $\partial P$ is the $(d-1)$-complex $\partial P=\bar{P}-\{P\}$.

Definition 4.1. An ordering $F_{1}, F_{2}, \ldots, F_{t}$ of the facets of a $d$-complex $\Delta$ is said to be a shelling if $d=0$ or if $d>0$ and for $j=2,3, \ldots, t, \bar{F}_{j} \cap \cup_{i=1}^{j-1} \bar{F}_{i}$ is a $(d-1)$ complex having a shelling which extends to a shelling of $\partial F_{j}$ (i.e., $\partial F_{j}$ has a shelling in which the facets of $\bar{F}_{j} \cap \cup_{i=1}^{j-1} \bar{F}_{i}$ come first). $\Delta$ is said to be shellable if it admits a shelling.

It is easy to see that when $\Delta$ is simplicial this recursive definition reduces to the usual nonrecursive definition of shellability stated in \$2. Similar but slightly less restrictive versions of shellability for $d$-complexes have been proposed by Bruggesser and Mani [3] and Danaraj and Klee [5].

Proposition 4.2 (BRUgGesser AND MANI). The boundary complex of a polytope is shellable.

Although in [3], Bruggesser and Mani present this result using a less restrictive version of shellability, their proof carries through for our version.

The face lattice $L(\Delta)$ of a $d$-complex $\Delta$ is obtained by ordering the faces of $\Delta$ by inclusion and adjoining a greatest element $\hat{1}$.

THEOREM 4.3. Let $\Delta$ be a d-complex. The face lattice $L(\Delta)$ admits a recursive coatom ordering if and only if $\Delta$ is shellable.

Proof. We will prove for all $d$ by induction that a recursive ordering of the coatoms of $L(\Delta)$ is a shelling order of the facets of $\Delta$, and conversely. This is clearly true for $d=0$. 
Assume that $d \geqslant 1$. Observe first that if $F_{1}, F_{2}, \ldots, F_{t}$ is any ordering of the coatoms of $L(\Delta)$ (i.e., the facets of $\Delta$ ) then Definition 3.1(ii) applied to the coatoms is equivalent to saying that $\bar{F}_{j} \cap \cup_{i=1}^{j-1} \bar{F}_{i}$ is a $(d-1)$-complex for $j=2, \ldots, t$.

Now suppose that $F_{1}, F_{2}, \ldots, F_{t}$ is a recursive coatom ordering. For any $j=2, \ldots, t$, the facets of $\bar{F}_{j} \cap \cup_{i=1}^{j-1} \bar{F}_{i}$ are precisely those coatoms of $\left[\hat{0}, F_{j}\right]$ which are covered by some $F_{i}, i<j$. It follows from Definition 3.1(i) that these coatoms come first in a recursive coatom ordering of $\left[\hat{0}, F_{j}\right]$. Since by induction this recursive coatom ordering of $\left[\hat{0}, F_{j}\right]$ is a shelling order of $\partial F_{j}$, we can conclude that $\bar{F}_{j} \cap \cup_{i=1}^{j-1} \bar{F}_{i}$ has a shelling which extends to a shelling of $\partial F_{j}$.

Conversely, let $F_{1}, F_{2}, \ldots, F_{t}$ be a shelling of $\Delta$. For any $j=2, \ldots, t$, since $\bar{F}_{j} \cap \cup_{i=1}^{j-1} \bar{F}_{i}$ has a shelling which extends to a shelling of $\partial F_{j}$, we can conclude by induction that $\left[\hat{0}, F_{j}\right]$ admits a recursive coatom ordering in which the facets of $\bar{F}_{j} \cap \cup_{i=1}^{j-1} \bar{F}_{i}$ are the coatoms that come first. These coatoms are precisely those that are covered by some $F_{i}, i<j$. Hence, part (i) of Definition 3.1 holds for $j=2, \ldots, t$. It follows from Proposition 4.2 that $\partial F_{1}$ is shellable, so also [0, $\left.F_{1}\right]$ admits a recursive coatom ordering.

COROLlaRY 4.4. The face lattice of a d-complex $\Delta$ is dual CL-shellable if and only if $\Delta$ is shellable.

Since there are several classes of simplicial complexes which are known to be shellable, Corollary 4.4 provides a wide variety of CL-shellable posets. For instance, finite Coxeter complexes, Tits buildings and matroid complexes are all examples of shellable simplicial complexes. Hence the face lattices of these complexes are dual CL-shellable.

THEOREM 4.5. The face lattice of a polytope is both CL-shellable and dual CL-shellable.

Proof. Dual CL-shellability is an immediate consequence of Corollary 4.4 and Proposition 4.2. The (order) dual of the face lattice of a polytope is isomorphic to the face lattice of the (polar) dual polytope. Hence, the face lattice is also CL-shellable.

5. Totally semimodular posets. A finite poset $P$ is said to be semimodular if it is bounded and whenever two distinct elements $u, v \in P$ both cover $x \in P$ there is a $z \in P$ which covers both $u$ and $v$. Semimodular posets are graded but not necessarily shellable. $P$ is said to be totally semimodular if it is bounded and all intervals $[x, y]$ are semimodular. Totally semimodular posets are known to be shellable $[1, \S 6]$, and will now be shown to be CL-shellable. Semimodular lattices are actually EL-shellable $[1, \S 3]$, but the question of EL-shellability for nonlattice totally semimodular posets remains open.

THEOREM 5.1. A graded poset $P$ is totally semimodular if and only if for every interval $[x, y]$ of $P$, every atom ordering in $[x, y]$ is a recursive atom ordering.

Proof. We prove this by induction on the length of $P$. For posets of length 1 , the result is trivial. 
Let $P$ be totally semimodular with length greater than 1 . If $[x, y] \neq P$ then $[x, y]$ is totally semimodular and by induction every atom ordering in $[x, y]$ is a recursive atom ordering.

Let $a_{1}, a_{2}, \ldots, a_{t}$ be any atom ordering in $P$. Since every atom ordering in $\left[a_{j}, \hat{1}\right]$ is recursive, order the atoms of $\left[a_{j}, \hat{1}\right]$ so that those that cover some $a_{i}, i<j$, come first. Let $y>a_{i}, a_{j}, i<j$. Since $P$ is totally semimodular there is an element $z \leqslant y$ which covers $a_{i}$ and $a_{j}$. Thus (i) and (ii) of Definition 3.1 are satisfied by $a_{1}, a_{2}, \ldots, a_{t}$, so this atom ordering is recursive.

Conversely, to show that $P$ is totally semimodular we must show that if $u, v, x, y$ $\in P$ where $x \rightarrow u, x \rightarrow v$ and $u, v<y$ then there is an element $z \leqslant y$ which covers $u$ and $v$. Order the atoms of $[x, y]$ so that $u$ and $v$ come first. Since every atom ordering of $[x, y]$ is recursive, it follows from Definition 3.1(ii) that $u$ and $v$ are covered by an element $z \leqslant y$.

COROLlary 5.2. A totally semimodular poset is CL-shellable.

6. Linguistic posets. Let $A$ be a finite alphabet, $|A| \geqslant 2$. By a word of length $k \geqslant 0$ is meant a string of $k$ letters drawn from $A$. Given two words $w_{1}$ and $w_{2}$ we say that $w_{1}$ is a subword of $w_{2}$, written $w_{1} \leqslant w_{2}$, if $w_{2}=a_{1} a_{2} \cdots a_{q}$ and $w_{1}=a_{i_{1}} a_{i_{2}} \cdots a_{i_{k}}$, $1 \leqslant i_{1}<i_{2}<\cdots<i_{k} \leqslant q$. Any set of words is partially ordered by the subword relation. A word is said to be injective if no letter occurs more than once, and normal if no two consecutive letters are equal. Let $I_{A}$ and $N_{A}$ denote the posets of all injective and normal words, respectively, and let $N_{A, k}$ denote the poset of all normal words of length at most $k . N_{A}$ is infinite but $I_{A}$ and $N_{A, k}$ are finite. By convention we exclude the empty word from these posets. Thus, $\hat{I}_{A}$ and $\hat{N}_{A, k}$ are graded posets of length $|A|+1$ and $k+1$, respectively, and the word length $l(w)$ coincides with the poset rank. The posets $I_{\{a, b, c\}}$ and $N_{\{a, b\}, 3}$ are depicted in Figure 6.1.
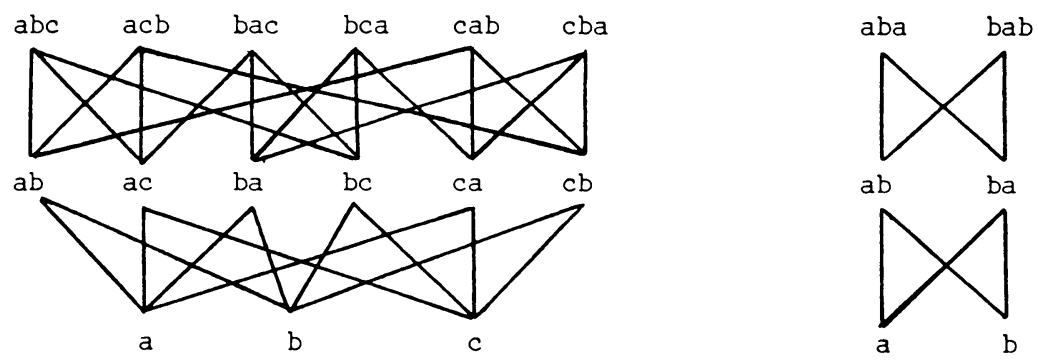

FIGURE 6.1

The linguistic posets $I_{A}$ and $N_{A, k}$ were introduced and studied by F. Farmer in [7].

THEOREM 6.1. (i) $\hat{I}_{A}$ is dual CL-shellable.

(ii) $\hat{N}_{A, k}$ is dual $C L$-shellable, for all $k \geqslant 1$.

The two parts of this result will be proved by quite different methods. For part (i) we will exhibit an explicit recursive coatom ordering, while part (ii) will be dealt with using Coxeter group methods. Before we turn to the proofs, let us briefly discuss some connections with Farmer's work. 
Suppose that $|A|=n$, and let $S \subseteq[n]$. Define $I_{A, S}=\left\{w \in I_{A} \mid l(w) \in S\right\}$, i.e., $I_{A, S}$ is the poset of injective words of length prescribed by $S$. We will derive an expression for the Möbius function $\mu\left(I_{A, S}\right)$, i.e., $\mu(\hat{0}, \hat{1})$ computed on $\hat{I}_{A, S}$, and discuss the homotopy type of $I_{A, S}$. For some basic facts concerning Möbius functions, see e.g. $[8, \S 5]$ and the further references found there. Given a sequence of integers $1 \leqslant k_{1}<k_{2}<\cdots<k_{i}$, let $\mathscr{D}\left(k_{1}, k_{2}, \ldots, k_{i}\right)$ denote the set of permutations of $\left[k_{i}\right]$ having descent set $\left\{k_{1}, k_{2}, \ldots, k_{i-1}\right\}$. In other words,

$$
\mathcal{D}\left(k_{1}, k_{2}, \ldots, k_{i}\right)=\left\{\pi \in S_{\left[k_{i}\right]} \mid \pi_{j}>\pi_{j+1} \Leftrightarrow j \in\left\{k_{1}, k_{2}, \ldots, k_{i-1}\right\}\right\} .
$$

Thus, $\left|\mathscr{D}\left(k_{1}\right)\right|=1$ and $|\mathscr{D}(1,2, \ldots, i)|=1$. Using the natural EL-labeling of the Boolean lattice $\mathscr{B}\left(k_{i}\right)$ of rank $k_{i}$, one sees from Stanley's formula [1, Theorem 2.7] that $(-1)^{i}\left|\mathscr{Q}\left(k_{1}, k_{2}, \ldots, k_{i}\right)\right|$ equals the Möbius function of the $\left\{k_{1}, k_{2}, \ldots, k_{i-1}\right\}-$ rank-selected subposet of $\mathscr{B}\left(k_{i}\right)$. Rank-selected subposets are defined in $\S 8$ below. Now, clearly every lower interval $[\hat{0}, w], w \neq \hat{1}$, in $\hat{I}_{A}$ is Boolean. Thus, if $S=$ $\left\{k_{1}, k_{2}, \ldots, k_{s}\right\}, 1 \leqslant k_{1}<k_{2}<\cdots<k_{s} \leqslant n$, we derive the following expression:

$$
\mu\left(I_{A, S}\right)=-1+\sum_{i=1}^{s}(-1)^{i+1}(n)_{k_{i}}\left|\mathscr{D}\left(k_{1}, k_{2}, \ldots, k_{i}\right)\right| .
$$

Here, $(n)_{k_{i}}=n(n-1) \cdots\left(n-k_{i}+1\right)$ is the number of injective words of length $k_{i}$.

Since rank-selection preserves shellability (cf. Theorem 8.1 below or [1, Theorem 4.1]) it follows from Theorem 6.1 that $I_{A, S}$ is shellable. A shellable poset is known to be homotopy Cohen-Macaulay (cf. [1, Appendix]). In particular, we may conclude that (the order complex of) $I_{A, S}$ has the homotopy type of a wedge of $\sum_{i=0}^{s}(-1)^{s+i}(n)_{k_{i}}\left|\mathcal{D}\left(k_{1}, k_{2}, \ldots, k_{i}\right)\right|(s-1)$-spheres. This was shown by Farmer for the case $S=\{1,2, \ldots, s\}, s \leqslant n$, cf. [7, Theorem 5 and Remark].

A similar discussion applies to the poset of normal words. Let $S$ be any finite set of positive integers and define $N_{A, S}=\left\{w \in N_{A} \mid l(w) \in S\right\}$. In the same way it follows from Theorem 6.1 that $N_{A, S}$ is shellable, and hence has the homotopy type of a wedge of $(|S|-1)$-spheres. Again, the $S=\{1,2, \ldots, s\}$ case is due to Farmer [7, Theorem 4]. He also obtains in [7, Remark, p. 611]:

$$
\mu\left(N_{A, k}\right)=-1+\sum_{i=1}^{k}(-1)^{i-1} n(n-1)^{i-1}=(-1)^{k-1}(n-1)^{k} .
$$

We will later derive a slight generalization of this.

Proof OF THEOREM 6.1(i). We will show that $\hat{I}_{A}$ admits a recursive coatom ordering. It is clear that the maximal words of $I_{A}$ are the permutations of $A$. Choose an ordering of $A$ and order the permutations lexicographically. We claim that this is a recursive coatom ordering of $\hat{I}_{A}$.

We need only establish part (ii) of Definition 3.1 since the intervals below the maximal words are Boolean, and by Theorem 5.1 every coatom ordering of a Boolean lattice is recursive. For each $w=a_{1} a_{2} \cdots a_{k}$ let $w^{\perp}$ be the word formed by the letters not in $w$ arranged in increasing order. There is a unique maximal word $\mathcal{E}(w)$ above $w$ and $w^{\perp}$ such that if $x$ is any letter in $w^{\perp}$ then $x$ is greater than all its 
predecessors and less than its immediate successor in $\mathcal{E}(w)$. For example, if $A=\{1,2, \ldots, 9\}$ and $w=4285$ then $w^{\perp}=13679$ and $\mathcal{E}(w)=134267859$. It is not difficult to verify that $\mathcal{E}(w)$ is the lexicographically first maximal word above $w$.

Now, suppose that $w<m_{i}, m_{j}$, where $m_{i}$ and $m_{j}$ are maximal words and $m_{i}$ precedes $m_{j}$ lexicographically. Then $m_{j} \neq \mathcal{E}(w)$. Thus there is a letter $x$ of $m_{j}$ and not in $w$ such that $x$ is less than a predecessor or greater than its immediate successor. Remove such an $x$ from $m_{j}$ to get the word $z \geqslant w$. By letting $m_{k}=\mathcal{E}(z)$ $\neq m_{j}$ we obtain what is needed to satisfy Definition 3.1(ii).

In the sequel the notion of a Coxeter group $(W, S)$, its Bruhat ordering and basic properties will be considered known. We adhere to the terminology and notation of $[2, \S 2]$, and readers desiring further details are advised to consult [2] and the references cited there.

Lemma 6.4. Let $(W, S)$ be a Coxeter group, $J \subseteq S$. Then $W^{J}$ is a directed poset, i.e., every two elements have a common upper bound.

Proof. Our principal tool will be the following lifting property, due to Verma [10, p. 395]: if $v, v^{\prime} \in W, s \in S$, and $v<v^{\prime}$, $v s>v$ and $v^{\prime} s<v^{\prime}$, then $v s \leqslant v^{\prime}$ and $v \leqslant v^{\prime} s$.

Suppose first that $w, w^{\prime} \in W$. We will prove that $U B\left(w, w^{\prime}\right)=\left\{u \in W \mid w, w^{\prime} \leqslant\right.$ $u\} \neq \varnothing$ by induction on $l(w)+l\left(w^{\prime}\right)$.

The case $l(w)+l\left(w^{\prime}\right) \leqslant 1$ is clear. In general, choose $s \in S$ so that $w s<w$. By induction there exists $u \in W$ such that $w s, w^{\prime} \leqslant u$. If $u s<u$, then by the lifting property $w \leqslant u$. If $u s>u$, then by the lifting property $w \leqslant u s$. Hence, in either case we are done.

Now suppose that $w, w^{\prime} \in W^{J}$. Let $u$ be a minimal element of $U B\left(w, w^{\prime}\right)$. If $u \notin W^{J}$ we can find $s \in J$ such that $u s<u$. Since $w s>w, w^{\prime} s>w^{\prime}$, the lifting property gives $w, w^{\prime} \leqslant u s$, which contradicts the minimality of $u$. Hence, $u \in W^{J}$.

When $W^{J}$ is finite the lemma amounts to the known fact that $W^{J}$ has a greatest element $w_{0}^{J}$. We will here however be primarily concerned with the case when $W^{J}$ is infinite.

Let $(W, S)$ be a Coxeter group, $|S|<\infty, J \subseteq S$, and let $Y$ be a finite set of positive integers. Define $\left(W^{J}\right)_{Y}=\left\{w \in W^{J} \mid l(w) \in Y\right\}$. Since $S$ and $Y$ are finite $\left(W^{J}\right)_{Y}$ is finite, and $\widehat{\left(W^{J}\right)_{Y}}$ is a graded poset.

TheOREM 6.5. $\widehat{\left(W^{J}\right)_{Y}}$ is dual CL-shellable.

Proof. It follows from the lemma by induction that $\left(W^{J}\right)_{Y}$ has an upper bound $u \in W^{J}$. Hence, $\widehat{\left(W^{J}\right)_{Y}}$ is simply the rank-selected subposet $[e, u]_{Y}^{J}$. Since $[e, u]^{J}$ is dual CL-shellable [2, Theorem 4.2], and this property is preserved under rank-selection (Theorem 8.1 below) the result follows.

Proof of Theorem 6.1(ii). Given the finite alphabet $A$, let $\left(W_{A}, A\right)$ be the Coxeter group whose Coxeter graph is the complete graph on vertex set $A$ with all edges labeled " $\infty$ ". Equivalently, $W_{A}$ is the group generated by $A$ subject only to the relations $a^{2}=e$ for all $a \in A$. Then every $w \in W_{A}$ clearly has a unique reduced 
expression, and this expression is a normal word in the alphabet $A$. Conversely, every normal word in $A$ is a reduced expression in $W_{A}$. Hence, $N_{A}$ can be identified with $W_{A}$, and because of the subword property (cf. $\left.[2,2.3]\right)$ the subword ordering of $N_{A}$ coincides with the Bruhat ordering of $W_{A}$. If $W=W_{A}, J=\varnothing$ and $Y=$ $\{1,2, \ldots, k\}$ then $\left(W^{J}\right)_{Y}=N_{A, k}$, so the result follows as a special case of Theorem 6.5 .

The proof suggests the following generalization. Given a finite alphabet $A$ and a nonempty subset $J \subseteq A$, let $N_{A, J, k}$ denote the poset of all normal words of length at most $k$ which end in a letter from $J$.

COROLlaRY 6.6. $\hat{N}_{A, J, k}$ is dual CL-shellable, for all $k \geqslant 1$.

This follows because, in the notation of the preceding proof, $N_{A, J, k}=\left(W^{A-J}\right)_{Y}$. If $w \in W^{A-J}$ then by the Deodhar-Verma theorem $\mu(e, w)=(-1)^{l(w)}$ if $[e, w]^{A-J}$ is full, and $=0$ otherwise (cf. [2,5.3]). In the particular Coxeter group $\left(W_{A}, A\right)$ it is easy to see that $[e, w]^{A-J}$ is full if and only if all letters in the unique reduced expression for $w$ come from $J$. Thus, if $|J|=j$, since there are $j(j-1)^{i-1}$ normal words in $J$ of length $i$, we obtain

$$
\mu\left(N_{A, J, k}\right)=-1+\sum_{i=1}^{k}(-1)^{i-1} j(j-1)^{i-1}=(-1)^{k-1}(j-1)^{k} .
$$

It can be shown that $N_{J, k}$ is a strong deformation retract of $N_{A, J, k}$, so (6.7) can also be deduced from Farmer's formula (6.3).

Finally we remark that the corresponding poset $I_{A, J}$ of injective words which end in a letter from $J, \varnothing \neq J \subseteq A$, is not necessarily shellable when $J \neq A$. For instance, if $A=\{a, b, c\}$ and $J=\{a, b\}$, then $I_{A, J}$ has the homotopy type of a circle.

7. Lattices of bilinear forms. Let $V$ and $W$ be finite-dimensional vector spaces over $G F(q)$. Consider the poset whose elements are $\{(A, f) \mid A$ is a subspace of $V$ and $f: A \rightarrow W$ is a linear mapping $\}$ and whose order relation is given by $(A, f) \leqslant(B, g)$ if $A \subseteq B$ and $g$ restricted to $A$ is $f$. This poset was suggested to us by D. Stanton, who calls it the (lower) semilattice of bilinear forms [9, p. 278]. Its definition goes back to work by Delsarte [6]. We adjoin a top element $\hat{1}$ to obtain a lattice $L_{q}(V, W)$. The lattice of bilinear forms $L_{q}(V, W)$ is clearly graded, and if $x=(A, f)$ then $\rho(x)=\operatorname{dim} A$ and the lower interval $[\hat{0}, x]$ is isomorphic to the lattice of subspaces of $A$. The crucial recursive property of $L_{q}(V, W)$ is that the upper interval $[x, \hat{1}]$ is itself isomorphic to a lattice of bilinear forms.

LemMa 7.1. Suppose $x=(A, f) \in L_{q}(V, W)$, and let $B$ be a complementary subspace to $A$, so that $V=A \oplus B$. Define a map $\varphi_{x, B}:[x, \hat{1}] \rightarrow L_{q}(B, W)$ by $\varphi(C, g)$ $=\left(C \cap B,\left.g\right|_{C \cap B}\right)$ and $\varphi(\hat{1})=\hat{1}$. Then $\varphi$ is a poset isomorphism.

Proof. The verification is straightforward and will be omitted.

TheOREM 7.2. $L_{q}(V, W)$ is $C L$-shellable.

Proof. Again we rely on the recursive formulation of CL-shellability (cf. §3). We prove the following assertion by induction on $n=\operatorname{dim} V: \operatorname{In} L_{q}(V, W)$ any atom 
ordering in which all the atoms of the form $(\langle u\rangle, 0)$ come first is a recursive atom ordering. Here and in the sequel, if $u_{1}, u_{2}, \ldots, u_{k} \in V$ then $\left\langle u_{1}, u_{2}, \ldots, u_{k}\right\rangle$ denotes their linear span in $V$.

The assertion is trivial for $n=1$. Let $n>1$ and assume that $\Omega$ is any atom ordering for which the atoms of the form $(\langle u\rangle, 0)$ come first. For each atom $x$ of $L_{q}(V, W)$, let $F(x)$ be the set of all atoms of $[x, \hat{1}]$ which cover some atom of $L_{q}(V, W)$ which precedes $x$ in $\Omega$. Let $G(x)$ be the set of atoms of $[x, \hat{1}]$ whose image under $\varphi_{x, B}$ is of the form $(\langle w\rangle, 0)$. The definition of $G(x)$ depends on how $B$ is chosen (cf. Lemma 7.1), but the following arguments are independent of that choice. We want to find an atom ordering of $[x, \hat{1}]$ for which (i) the atoms in $F(x)$ come before the atoms that are not in $F(x)$ and (ii) the atoms in $G(x)$ come before the atoms that are not in $G(x)$.

Case 1. Assume that $x$ is of the form $(\langle u\rangle, 0)$. We claim that in this case $F(x) \subseteq G(x)$. If $(C, g) \in F(x)$ then $C$ contains a vector $v$ for which $g(v)=0$ and $\langle v\rangle \neq\langle u\rangle$. This implies that $g=0$ since $v$ and $u$ generate $C$. Now we have that $\varphi_{x, B}(C, g)=(C \cap B, 0)$ and hence $(C, g) \in G(x)$. Thus $F(x) \subseteq G(x)$.

Case 2. Assume that $x$ is not of the form $(\langle u\rangle, 0)$. This time we assert that $G(x) \subseteq F(x)$. If $(C, g) \in G(x)$ then $\varphi_{x, B}(C, g)=(\langle w\rangle, 0)$ where $\langle w\rangle=C \cap B$. Hence $(\langle w\rangle, 0)$ precedes $x$ in $\Omega$. Since $(\langle w\rangle, 0)<(C, g)$ it follows that $(C, g) \in F(x)$. Thus $G(x) \subseteq F(x)$.

For Case 1, we can order the atoms of $[x, \hat{1}]$ so that the atoms of $F(x)$ come first, then the atoms of $G(x)-F(x)$, and finally the remaining atoms. Similarly, for Case 2, we can order the atoms so that $G(x)$ comes first, then $F(x)-G(x)$, and finally the remaining atoms. Therefore in both cases it is possible to order the atoms of [ $x, \hat{1}]$ so that those in $F(x)$ precede those not in $F(x)$ and those in $G(x)$ precede those not in $G(x)$. Consequently by the induction hypothesis and Lemma 7.1, this atom ordering is recursive and Definition 3.1(i) is satisfied.

To complete the proof that $\Omega$ is a recursive atom ordering it remains only to verify part (ii) of Definition 3.1. Let $x_{i}=(\langle u\rangle, f)$ and $x_{j}=\left(\left\langle u^{\prime}\right\rangle, f^{\prime}\right)$ be atoms in $L_{q}(V, W)$, and suppose that $x_{i}$ precedes $x_{j}$ in $\Omega$.

Case $1 .\langle u\rangle=\left\langle u^{\prime}\right\rangle$ and $f \neq f^{\prime}$. If $x_{i}, x_{j}<y$, then clearly $y=\hat{1}$. Since $x_{i}$ precedes $x_{j}, f^{\prime} \neq 0$. Now let $x_{k}=\left(\left\langle u^{\prime \prime}\right\rangle, 0\right)$ where $\left\langle u^{\prime \prime}\right\rangle \neq\left\langle u^{\prime}\right\rangle$ and let $z=\left(\left\langle u^{\prime}, u^{\prime \prime}\right\rangle, g\right)$ where $g\left(u^{\prime}\right)=f^{\prime}\left(u^{\prime}\right)$ and $g\left(u^{\prime \prime}\right)=0$. Then $x_{k}$ precedes $x_{j}$ in $\Omega$ and $x_{k}, x_{j} \rightarrow z \leqslant y$.

Case 2. $\langle u\rangle \neq\left\langle u^{\prime}\right\rangle$. If $x_{i}, x_{j}<y=(C, h)$, then $u, u^{\prime} \in C, h(u)=f(u)$ and $h\left(u^{\prime}\right)=f^{\prime}\left(u^{\prime}\right)$. Let $z=\left(\left\langle u, u^{\prime}\right\rangle, g\right)$ where $g(u)=f(u)$ and $g\left(u^{\prime}\right)=f^{\prime}\left(u^{\prime}\right)$. Then $x_{i}, x_{j} \rightarrow z \leqslant y$.

Let $X$ and $Y$ be finite nonempty sets, and consider the poset $P=\{(A, f) \mid \varnothing \neq$ $A \subseteq X, f: A \rightarrow Y\}$ with order relation $(A, f) \leqslant(B, g)$ if $A \subseteq B$ and $\left.g\right|_{A}=f$. Then $L(X, Y)=\hat{P}$ is a lattice. A simple modification of the proof of Theorem 7.2 yields the following.

\section{THEOREM 7.3. $L(X, Y)$ is CL-shellable.}

It is also possible to show that $L(X, Y)$ is dual CL-shellable. We omit the details. Figure 7.1(a) shows the proper part of $L([2],[2])$. As an aside, consider the subposet 
$I L(X, Y)$ consisting of $\hat{0}, \hat{i}$ and all $(A, f)$ in $L(X, Y)$ such that $f$ is injective. $I L(X, Y)$ is a lattice, and actually a sub-lower-semilattice of $L(X, Y)$. The lattices $I L([n],[n])$ have been investigated by Cameron and Deza [4] under the name of permutation geometries. Unfortunately, injectivity here seems to destroy the shellability property, as can be seen from $I L([2],[2])$, the proper part of which is depicted in Figure 7.1(b).

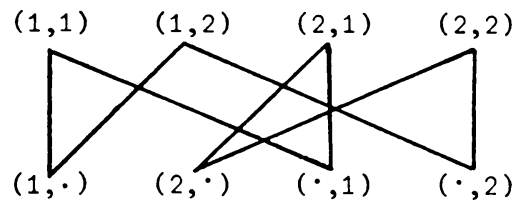

(a)

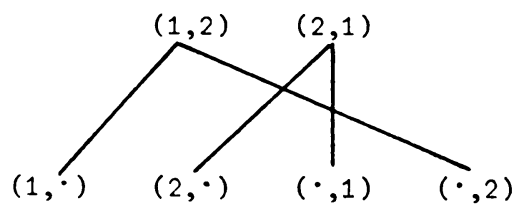

(b)

FigURE 7.1

Suppose now that $|X|=n,|Y|=m$, and let $S=\left\{k_{1}, k_{2}, \ldots, k_{s}\right\}, 1 \leqslant k_{1}<k_{2}<$ $\cdots<k_{s} \leqslant n$. Consider $\mu\left(L(X, Y)_{S}\right)$, i.e., the Möbius function $\mu(\hat{0}, \hat{1})$ computed on the rank-selected subposet $L(X, Y)_{S}=\{(A, f) \in L(X, Y) \| A \mid \in S\} \cup\{\hat{0}, \hat{1}\}$. Since there are $\left(\begin{array}{c}n \\ k_{i}\end{array}\right) m^{k_{i}}$ elements of rank $k_{i}$ in $L(X, Y)$ and if $x$ is one of them then $\mu(\hat{0}, x)=(-1)^{i}\left|\mathscr{D}\left(k_{1}, k_{2}, \ldots, k_{i}\right)\right|$ computed in $L(X, Y)_{S}$ (cf. the discussion preceding formula (6.2)), we obtain the following expression:

$$
\mu\left(L(X, Y)_{S}\right)=-1+\sum_{i=1}^{s}(-1)^{i+1}\left(\begin{array}{c}
n \\
k_{i}
\end{array}\right) m^{k_{i}}\left|\mathscr{D}\left(k_{1}, k_{2}, \ldots, k_{i}\right)\right| .
$$

A similar formula exists for the lattice $L_{q}(V, W)$ of bilinear forms. Suppose that $\operatorname{dim} V=n, \operatorname{dim} W=m$, and let $S$ be as before. There are $\left[\begin{array}{l}n \\ k_{i}\end{array}\right]_{q} q^{m k_{i}}$ elements of rank $k_{i}$ in $L_{q}(V, W)$, where $\left.\left[\begin{array}{l}n \\ k_{i}\end{array}\right]\right]_{q}$ denotes the Gaussian coefficient

$$
\left(q^{n}-1\right)\left(q^{n-1}-1\right) \cdots\left(q^{n-k_{i}+1}-1\right) /(q-1)\left(q^{2}-1\right) \cdots\left(q^{k_{i}}-1\right) .
$$

Furthermore, if $\rho(x)=k_{i}$ then $[\hat{0}, x]$ is isomorphic to the subspace lattice of a $k_{i}$-dimensional space over $G F(q)$. Hence, by a formula of Stanley [8, p. 155] the Möbius function $\mu(\hat{0}, x)$ computed in $L_{q}(V, W)_{S}=\left\{(A, f) \in L_{q}(V, W) \mid \operatorname{dim} A \in\right.$ $S\} \cup\{\hat{0}, \hat{1}\}$ is $\mu(\hat{0}, x)=(-1)^{i} \Sigma_{\pi} q^{\operatorname{inv}(\pi)}$, the sum extending over all permutations $\pi \in \mathscr{D}\left(k_{1}, k_{2}, \ldots, k_{i}\right)$ and $\operatorname{inv}(\pi)$ denoting the number of inversions in $\pi$. We deduce

$$
\mu\left(L_{q}(V, W)_{S}\right)=-1+\sum_{i=1}^{s}(-1)^{i+1}\left[\begin{array}{l}
n \\
k_{i}
\end{array}\right]_{q} q^{m k_{i}} \sum_{\pi \in \mathcal{D}_{\left(k_{1}, k_{2}, \ldots, k_{i}\right)}} q^{\mathrm{inv}(\pi)} .
$$

8. Operations that preserve shellability. In $[1, \S 4]$ poset operations that preserve either shellability or EL-shellability are considered. In this section we extend all the results of $[1, \S 4]$ to both shellability and CL-shellability. The operations considered are rank-selection, direct products, ordinal sums, cardinal powers and interval posets. 
Let $P$ be a graded poset of length $n$ and with rank function $\rho$. For any $S \subseteq[n-1]$ the rank-selected subposet, $P_{S}$, is defined to be $P_{S}=\{x \in P \mid \rho(x) \in S$ $\cup\{0, n\}\}$.

Let $P$ and $Q$ be posets. The direct product $P \times Q$ is the poset defined on the product set by $(x, y) \leqslant\left(x^{\prime}, y^{\prime}\right)$ if $x \leqslant x^{\prime}$ in $P$ and $y \leqslant y^{\prime}$ in $Q$. The ordinal sum $P \oplus Q$ is the poset defined on the disjoint union of $P$ and $Q$ by $x \leqslant y$ in $P \oplus Q$ if (i) $x, y \in P$ and $x \leqslant y$ in $P$, (ii) $x, y \in Q$ and $x \leqslant y$ in $Q$, or (iii) $x \in P$ and $y \in Q$. The cardinal power $Q^{P}$ is the set of orderpreserving maps $f: P \rightarrow Q$, partially ordered by $f \leqslant g$ if $f(x) \leqslant g(x)$ for all $x \in P$.

The interval poset $\operatorname{Int}(P)$ of a poset $P$ is the set of intervals (including the empty interval) ordered by containment.

In [1, Theorem 4.1] it is shown that rank-selection preserves shellability. We will prove the corresponding result for CL-shellability. Whether rank-selection preserves EL-shellability remains open.

THEOREM 8.1. If $P$ is a CL-shellable poset of rank $n$ then $P_{S}$ is $C L$-shellable for all $S \subseteq[n-1]$.

Proof. We shall prove the result for $S=[n-1]-\{r\}$ where $r \in[n-1]$. The general result follows by induction. Let $\lambda: \mathcal{E}^{*}(P) \rightarrow \Lambda$ be a CL-labeling of $P$. Define a labeling $\lambda_{S}: \mathcal{E}^{*}\left(P_{S}\right) \rightarrow \Lambda \times \Lambda$ as follows. If $c=\left(\hat{0}=x_{0} \rightarrow x_{1} \rightarrow \cdots \rightarrow x_{r-1} \rightarrow\right.$ $\left.x_{r+1} \rightarrow \cdots \rightarrow x_{n}=\hat{1}\right)$ is a maximal chain in $P_{S}$ and if $x_{r}$ is the element of rank $r$ on the lexicographically first maximal chain in the rooted interval $\left(\left[x_{r-1}, x_{r+1}\right], 0 \rightarrow x_{1}\right.$ $\left.\rightarrow \cdots \rightarrow x_{r-1}\right)$ of $P$ then let

$$
\begin{aligned}
& \lambda_{S}\left(c, x_{i-1}, x_{i}\right)=\left(\lambda\left(c \cup x_{r}, x_{i-1}, x_{i}\right), \lambda\left(c \cup x_{r}, x_{i-1}, x_{i}\right)\right) \text { if } i=1, \ldots, r-1, \\
& \lambda_{S}\left(c, x_{r-1}, x_{r+1}\right)=\left(\lambda\left(c \cup x_{r}, x_{r-1}, x_{r}\right), \lambda\left(c \cup x_{r}, x_{r}, x_{r+1}\right)\right),
\end{aligned}
$$

and

$$
\lambda_{S}\left(c, x_{i-1}, x_{i}\right)=\left(\lambda\left(c \cup x_{r}, x_{r-1}, x_{r}\right), \lambda\left(c \cup x_{r}, x_{i-1}, x_{i}\right)\right) \text { if } i=r+2, \ldots, n .
$$

Now order $\Lambda \times \Lambda$ lexicographically (this ordering is stronger than direct product order). It is then straightforward to verify that $\lambda_{S}$ is a CL-labeling of $P_{S}$.

Before considering the remaining operations we need to recall the following fact, whose proof can be found in [1, Proposition 4.2].

Proposition 8.2. If $P$ is a shellable poset then all intervals of $P$ are shellable.

In [1, Theorem 4.3] it is shown that direct products preserve EL-shellability. The corresponding result is true also for CL-shellable and shellable bounded posets. For CL-shellable posets the proof in [1] requires no significant modification. For shellable posets we have the following.

ThEOREM 8.3. Let $P$ and $Q$ be bounded finite posets. Then $P \times Q$ is shellable if and only if both $P$ and $Q$ are shellable.

Proof. The product $P \times Q$ is clearly graded if and only if both $P$ and $Q$ are graded. By Proposition 8.2 since $P \cong[(\hat{0}, \hat{0}),(\hat{1}, \hat{0})]$ and $Q \cong[(\hat{0}, \hat{0}),(\hat{0}, \hat{1})], P$ and $Q$ are shellable if $P \times Q$ is shellable. 
Suppose that $P$ and $Q$ are bounded shellable posets of lengths $m$ and $n$ respectively. The length of $P \times Q$ is then $m+n$. A covering relation $(x, y) \rightarrow\left(x^{\prime}, y^{\prime}\right)$ occurs in $P \times Q$ if and only if $x=x^{\prime}$ and $y \rightarrow y^{\prime}$ or $x \rightarrow x^{\prime}$ and $y=y^{\prime}$. The edges of $P \times Q$ can therefore be labeled as follows:

$$
\lambda\left((x, y),\left(x^{\prime}, y^{\prime}\right)\right)= \begin{cases}0 & \text { if } y=y^{\prime} \\ 1 & \text { if } x=x^{\prime}\end{cases}
$$

Now, corresponding to each maximal chain $c=\left(\hat{0}=z_{0} \rightarrow z_{1} \rightarrow \cdots \rightarrow z_{m+n}=\hat{1}\right)$ there is an $(n+m)$-tuple $\sigma(c)$ of $m$ 0's and $n$ l's defined by

$$
\sigma(C)=\left(\lambda\left(\hat{0}, z_{1}\right), \lambda\left(z_{1}, z_{2}\right), \ldots, \lambda\left(z_{m+n-1}, z_{m+n}\right)\right) .
$$

We define two projection maps $\Pi_{P}: \mathfrak{N}(P \times Q) \rightarrow \mathfrak{N}(P)$ and $\Pi_{Q}: \mathfrak{N}(P \times Q)$ $\rightarrow \mathfrak{T}(Q)$ by $\Pi_{P}(c)=\left\{x_{i} \mid i=0,1, \ldots, m+n\right\}$ and $\Pi_{Q}(c)=\left\{y_{i} \mid i=0,1, \ldots, m+\right.$ $n\}$ for $c=\left(\hat{0}=\left(x_{0}, y_{0}\right) \rightarrow\left(x_{1}, y_{1}\right) \rightarrow \cdots \rightarrow\left(x_{m+n}, y_{m+n}\right)=\hat{1}\right)$.

Each maximal chain $c$ in $P \times Q$ can now be uniquely represented by the triple $\left(\sigma(c), \Pi_{P}(c), \Pi_{Q}(c)\right)$. If $\Omega_{P}$ and $\Omega_{Q}$ are the shelling orders of $P$ and $Q$, respectively, then the triples can be lexicographically ordered by using the lexicographical ordering, denoted by $<_{L}$, on the $(n+m)$-tuples of 0's and 1's and the shelling orders $\Omega_{P}$ and $\Omega_{Q}$ on $\left\{\Pi_{P}(c) \mid c \in \Re(P \times Q)\right\}=\Re(P)$ and $\left\{\Pi_{Q}(c) \mid c \in\right.$ $\mathfrak{K}(P \times Q)\}=\mathfrak{R}(Q)$, respectively. Let $\Omega$ be the induced linear ordering of $\mathfrak{T}(P \times Q)$.

We now show that $\Omega$ is a shelling order of $\mathscr{N}(P \times Q)$ by establishing Property $\mathrm{S}$. Suppose that $c=\left(\hat{0}=z_{0} \rightarrow z_{1} \rightarrow \cdots \rightarrow z_{m+n}=\hat{1}\right)$ and $c^{\prime}=\left(\hat{0}=z_{0}^{\prime} \rightarrow z_{1}^{\prime} \rightarrow \cdots \rightarrow\right.$ $\left.z_{m+n}^{\prime}=\hat{1}\right)$ are two maximal chains of $P \times Q$ such that $c^{\prime}<{ }^{\Omega} c$. Let $T=\{t \in$ $\left.[m+n] \mid z_{t} \neq z_{t}^{\prime}\right\}$. There are two cases to consider.

Case 1. Assume that $\sigma(c)$ has a descent at $t \in T$, i.e., $\lambda\left(z_{t-1}, z_{t}\right)>\lambda\left(z_{t}, z_{t+1}\right)$. Let $z_{t-1}=\left(a_{1}, a_{2}\right)$ and $z_{t+1}=\left(b_{1}, b_{2}\right)$. Since there is a descent at $t, z_{t}=\left(a_{1}, b_{2}\right)$. Let $c^{\prime \prime}=c-\left\{z_{t}\right\} \cup\left\{\left(b_{1}, a_{2}\right)\right\}$. Clearly $c^{\prime \prime}$ is a chain of $P \times Q$ and $\sigma\left(c^{\prime \prime}\right)<_{L} \sigma(C)$, which implies that $c^{\prime \prime}<^{\Omega} c$. It is also clear that $c^{\prime} \cap c \subseteq c^{\prime \prime} \cap c$ since $t \in T$.

Case 2. Assume that $\sigma(c)$ has no descents in $T$. It is not difficult to see that in this case $\sigma\left(c^{\prime}\right)=\sigma(c)$, since $c^{\prime}<^{\Omega} c$. We will show that $\sigma(c)$ cannot have ascents in $T$ either. (By having an ascent in $T$ we mean that $\lambda\left(z_{t-1}, z_{t}\right)<\lambda\left(z_{t}, z_{t+1}\right)$ for some $t \in T$.)

Suppose that $\sigma(c)$ has an ascent at $t \in T$. Let $l$ be the largest integer and $u$ the smallest integer such that $l<t<u$ and $l, u \in[m+n]-T$. Clearly, $0 \leqslant l<u \leqslant m$ $+n$ since $z_{0}^{\prime}=z_{0}$ and $z_{m+n}^{\prime}=z_{m+n}$. Let $z_{l}=\left(a_{1}, a_{2}\right)$ and $z_{u}=\left(b_{1}, b_{2}\right)$. Since there are no descents in $\sigma(c)$ between $l$ and $u$ and there is an ascent at $t, z_{t}=\left(b_{1}, a_{2}\right)$. Since $\sigma\left(c^{\prime}\right)=\sigma(c), z_{l}^{\prime}=z_{l}$, and $z_{u}^{\prime}=z_{u}$, we also have that $z_{t}^{\prime}=\left(b_{1}, a_{2}\right)$. Hence $z_{t}=z_{t}^{\prime}$, which contradicts the assumption that $t \in T$. Thus there are no ascents of $\sigma(c)$ in $T$.

Since $\sigma(c)=\sigma\left(c^{\prime}\right)$, either $\Pi_{P}\left(c^{\prime}\right)<\Omega_{P} \Pi_{P}(c)$ or $\Pi_{Q}\left(c^{\prime}\right)<\Omega_{Q} \Pi_{Q}(c)$. Without loss of generality we can assume the former. Let $\gamma$ be a maximal chain of $P$ such that $\gamma<{ }^{\Omega_{P}} \Pi_{P}(c)$ and $\Pi_{P}\left(c^{\prime}\right) \cap \Pi_{P}(c) \subseteq \gamma \cap \Pi_{P}(c)=\Pi_{P}(c)-\{x\}$ where $x \in \Pi_{P}(c)$. Let $u$ be the element on $\Pi_{P}(c)$ that is covered by $x$ and let $t \in[m+n]$ be such that $z_{t}=(x, y)$ and $z_{t-1}=(u, y)$ for some $y \in Q$. Clearly $z_{t}^{\prime} \neq z_{t}$ since $x \notin \Pi_{P}\left(c^{\prime}\right)$. This 
implies that $t \in T$, which in turn implies that $\sigma(c)$ has no ascent (or descent) at $t$. Hence, by letting $c^{\prime \prime}$ be the maximal chain of $P \times Q$ represented by the triple $\left(\sigma(c), \gamma, \Pi_{Q}(c)\right)$, we have that $c^{\prime \prime} \cap c=c-\{(x, y)\}=c-\left\{z_{t}\right\}$. It follows that $c^{\prime} \cap c \subseteq c^{\prime \prime} \cap c$ and $c^{\prime \prime}<^{\Omega} c$.

Theorem 8.3 is not stated in maximum generality. For the proof to go through it suffices to assume merely that $P$ has a greatest element and $Q$ a least element. If we instead assume that both $P$ and $Q$ have least elements, then the "only if" part holds, and we expect that the "if" part also holds but leave this open. Notice that if $P$ and $Q$ are shellable posets, one of which is nonacyclic, then $P \times Q$ is in general not shellable for topological reasons. For instance, let $P$ and $Q$ be the two shellable posets of Figure 8.1(a) and (b). The direct product $P \times Q$ is depicted in Figure $8.1(\mathrm{c})$, and its order complex $\Delta(P \times Q)$ triangulates the topological product of a circle and an interval, so it is a two-dimensional complex having the homotopy type of a circle. Such a complex cannot be shellable. Another quick way to see that this $P \times Q$ cannot be shellable is to compute the Möbius function $\mu(\hat{0}, x)$ in $(P \times Q)$ and see that it fails to satisfy the necessary condition $(-1)^{\rho(x)} \mu(\hat{0}, x) \geqslant 0$ for $x=\hat{1}$.

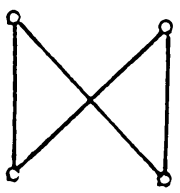

(a)

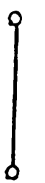

(b)

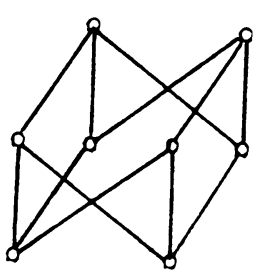

(c)

FIGURE 8.1

In [1, Theorem 4.5] it is shown that cardinal powers preserve EL-shellability of the base poset. Again, the extension of this result to CL-shellable posets is routine. We will prove the extension to shellable posets.

THEOREM 8.4. Let $Q$ be a finite poset. Then $P^{Q}$ is a shellable bounded poset if and only if $P$ is a shellable bounded poset.

Proof. First note that $P^{Q}$ is graded if and only if $P$ is graded. By Proposition 8.2, if $P^{Q}$ is shellable and bounded then $P$ is shellable and bounded, since $P \cong[f, g]$ in $P^{Q}$, where $f(x)=\hat{0}$ for all $x \in Q$ and $g(x)=\hat{0}$ for all $x \in Q$ with the exception of a maximal element $q \in Q$ for which $g(q)=\hat{1}$.

We prove the converse by induction on the cardinality of $Q$. It clearly holds for $|Q|=1$. It is not difficult to verify that if $q$ is any element of $Q$ then $P^{Q-\{q\}} \times P$ is isomorphic to the poset of maps from $Q$ to $P$ which are orderpreserving everywhere in $Q$ except at $q$, ordered by $f \leqslant g$ if $f(x) \leqslant g(x)$ for all $x \in Q$. Hence $P^{Q}$ is a subposet of $P^{Q^{-}\{q\}} \times P$. By induction and Theorem 8.3 we have that $P^{Q-\{q\}} \times P$ is shellable.

Let $q$ be a minimal element of $Q$ and let $\Omega$ be the shelling order of $P^{Q-\{q\}} \times P$ described in the proof of Theorem 8.3. Clearly the maximal chains of $P^{Q}$ are 
maximal chains of $P^{Q-\{q\}} \times P$. To show that $P^{Q}$ is shellable we will show that $\Omega$ restricted to $P^{Q}$ is a shelling. To do this we need only show that if $c_{1} \in \mathfrak{R}\left(P^{Q}\right)$, $c_{2} \in \mathfrak{R}\left(P^{Q-\{q\}} \times P\right), c_{2}<^{\Omega} c_{1}$, and $\left|c_{1} \cap c_{2}\right|=\left|c_{1}\right|-1$, then $c_{2} \in \Re\left(P^{Q}\right)$.

Let $c_{1}=\left(\hat{0}=f_{0} \rightarrow f_{1} \rightarrow \cdots \rightarrow f_{m}=\hat{1}\right)$ be a maximal chain of $P^{Q}$ and let $c_{2}=c_{1}$ $-\left\{f_{t}\right\} \cup\{g\}$ be a maximal chain of $P^{Q-\{q\}} \times P$ such that $c_{2}<{ }^{\Omega} c_{1}$. We must show that $g$ is orderpreserving on all of $Q$ in order to show that $c_{2} \in \Re\left(P^{Q}\right)$. Since $g \in P^{Q-\{q\}} \times P$ and $q$ is minimal we need only show that $g(x) \geqslant g(q)$ for all $x>q$.

Recall the labeling $\lambda$ of edges of $P^{Q-\{q\}} \times P$ described in the proof of Theorem 8.3: For $d \rightarrow e$ in $P^{Q-\{q\}} \times P$

$$
\lambda(d, e)= \begin{cases}0 & \text { if } d(q)=e(q), \\ 1 & \text { if } d(q) \rightarrow e(q) .\end{cases}
$$

Also, recall that $\sigma\left(c_{1}\right)=\left(\lambda\left(f_{0}, f_{1}\right), \lambda\left(f_{1}, f_{2}\right), \ldots, \lambda\left(f_{m-1}, f_{m}\right)\right)$. To show that $g(q) \leqslant$ $g(x)$ for all $x>q$, we need to consider 2 cases.

Case 1. Suppose $\lambda\left(f_{t-1}, g\right)=0$. This means that $f_{t-1}(q)=g(q)$. Hence if $g<x$ then $g(q)=f_{t-1}(q) \leqslant f_{t-1}(x) \leqslant g(x)$, since $f_{t-1}$ is orderpreserving and is less than $g$.

Case 2. Suppose $\lambda\left(f_{t-1}, g\right)=1$. We will show that in this case $\lambda\left(g, f_{t+1}\right)=1$ also. Assume $\lambda\left(g, f_{t+1}\right)=0$. It follows that $f_{t-1}(q) \rightarrow g(q)=f_{t+1}(q)$. Consequently $f_{t-1}(q)=f_{t}(q) \rightarrow f_{t+1}(q)$. This means that $\lambda\left(f_{t-1}, f_{t}\right)=0$ which implies that $\sigma\left(c_{1}\right)$ $<_{L} \sigma\left(c_{2}\right)$. Since this contradicts $c_{2}<^{\Omega} c_{1}$, we can conclude that $\lambda\left(g, f_{t+1}\right)=1$. Thus we now have that $g(q) \rightarrow f_{t+1}(q)$. Hence if $q<x$ then $g(q)<f_{t+1}(q) \leqslant f_{t+1}(x)=$ $g(x)$.

We now extend [1, Theorem 4.6] which deals with interval posets. Again, the extension to CL-shellable posets requires no significant modification.

Theorem 8.5. The poset $\operatorname{Int}(P)$ is a bounded shellable poset if and only if $P$ is a bounded shellable poset.

Proof. $\operatorname{Int}(P)$ is clearly graded if and only if $P$ is graded. Since $P \cong[[\hat{0}, \hat{0}],[\hat{0}, \hat{1}]]$, $P$ is shellable if $\operatorname{Int}(P)$ is shellable by Proposition 8.2.

To establish the converse, note that the elements of $\operatorname{Int}(P)$ of rank 1 are of the form $[x, x]$ where $x \in P$. We consider the intervals of $\operatorname{Int}(P)$ above the rank 1 elements, $[[x, x],[\hat{0}, \hat{1}]]$. Since these intervals are isomorphic to the direct product $[\hat{0}, x]^{*} \times[x, \hat{1}]$, they are shellable by Theorem 8.3 and Proposition 8.2 .

The shelling of $[[x, x],[\hat{0}, \hat{1}]]$ induces an ordering $\Omega_{x}$ of $\mathfrak{N}_{x}$, the set of maximal chains of $\operatorname{Int}(P)$ that contain $[x, x]$. By extending the partial ordering of $P$ to a linear ordering, $x_{1}, x_{2}, \ldots, x_{k}$, and letting the chains of $\Re_{x_{i}}$ ordered by $\Omega_{x_{i}}$, precede the chains of $\Re_{x_{j}}$, ordered by $\Omega_{x_{j}}$, for all $i<j$, we obtain a linear ordering $\Omega$ of TR $(\operatorname{Int}(P))$.

We now establish Property $S$ for $\Omega$. Let $c$ and $c^{\prime}$ be maximal chains of $\operatorname{Int}(P)$ such that $c^{\prime}<\Omega$. Let $c \in \mathfrak{K}_{x}$ and $c^{\prime} \in \mathfrak{R}_{x^{\prime}}$. If $x=x^{\prime}$ then we are done since the restiction $\Omega_{x}$ of $\Omega$ to $\Re_{x}$ is a shelling.

Now assume $x \neq x^{\prime}$. Let $[u, v]$ be the smallest common element of $c$ and $c^{\prime}$ that is greater than $\varnothing$, the empty interval. If $c$ is not the first chain in the ordering $\Omega$ that contains $[x, x],[u, v]$ and every element of $c$ greater than $[u, v]$, then by the 
shellability of $[[x, x],[\hat{0}, \hat{1}]]$ there is a $c^{\prime \prime} \in \mathscr{N}_{x}$ such that $c^{\prime \prime}<{ }^{\Omega} c$ and $c^{\prime} \cap c \subseteq c^{\prime \prime} \cap$ $c=c-\{z\}$ where $z \in[[x, x],[u, v]]$.

Assume that $c$ is the first chain in $\Re_{x}$ that contains $[u, v]$ and every element of $c$ greater than $[u, v]$. Since $\left[x^{\prime}, x^{\prime}\right]<[u, v]$ and $[x, x]<[u, v]$, it follows that $u \leqslant x$, $x^{\prime}$. If $u=x$ then $x \leqslant x^{\prime}$, which contradicts the fact that $x^{\prime}$ precedes $x$ in the linear extension of $P$. Therefore, $u<x$. Recall that the ordering $\Omega_{x}$ of $\mathfrak{N}_{x}$ corresponds to the shelling order of $[\hat{0}, x]^{*} \times[x, \hat{1}]$ which was described in the proof of Theorem 8.3. This ordering and the fact that $u<x$ implies that the element that covers $[x, x]$ on $c$ must be $[y, x]$ where $y \rightarrow x$ and $y \geqslant u$. Clearly the chains of $\Re_{y}$ precede the chains of $\mathfrak{R}_{x}$ in $\Omega$. If $c^{\prime \prime}=c-\{[x, x]\} \cup\{[y, y]\}$ then $c^{\prime \prime} \in \Re_{y}$ and hence $c^{\prime \prime}<^{\Omega} c$. It is also clear that $c^{\prime} \cap c \subseteq c^{\prime \prime} \cap c=c-\{[x, x]\}$.

In [1, Theorem 4.4] it is shown that ordinal sums preserve shellability and EL-shellability. This result extends easily to CL-shellable posets. Because of Theorem 8.1 we can in fact state a more complete result for CL-shellable posets than for EL-shellable posets.

THEOREM 8.6. The ordinal sum $\widehat{P \oplus Q}$ is CL-shellable if and only if both $\hat{P}$ and $\hat{Q}$ are CL-shellable.

9. Note added in proof. It is a consequence of Corollary 4.4 that if $\Delta$ is an unshellable complex whose barycentric subdivision is shellable, then the face lattice of $\Delta$ is shellable but not dual CL-shellable. Using this idea, A. Vince and M. Wachs and (independently) J. Walker have constructed shellable posets which are not CL-shellable. It is not known to us whether there exist CL-shellable posets which fail to be EL-shellable.

\section{REFERENCES}

1. A. Björner, Shellable and Cohen-Macaulay partially ordered sets, Trans. Amer. Math. Soc. 260 (1980), 159-183.

2. A. Björner and M. Wachs, Bruhat order of Coxeter groups and shellability, Adv. in Math. 43 (1982), 87-100.

3. H. Bruggesser and P. Mani, Shellable decompositions of cells and spheres, Math. Scand. 29 (1971), 197-205.

4. P. J. Cameron and M. Deza, On permutation geometries, J. London Math. Soc. (2) 20 (1979), $373-386$.

5. G. Danaraj and V. Klee, Shellings of spheres and polytopes, Duke Math. J. 41 (1974), 443-451.

6. P. Delsarte, Association schemes and t-designs in regular semilattices, J. Combin. Theory Ser. A 20 (1976), 230-243.

7. F. D. Farmer, Cellular homology for posets, Math. Japon. 23 (1979), 607-613.

8. R. P. Stanley, Balanced Cohen-Macaulay complexes, Trans. Amer. Math. Soc. 249 (1979), 139-157.

9. D. Stanton, A partially ordered set and $q$-Krawtchouk polynomials, J. Combin. Theory Ser. A 30 (1981), 276-284.

10. D.-N. Verma, Möbius inversion for the Bruhat ordering on a Weyl group, Ann. Sci. École Norm. Sup. 4 (1971), 393-398.

Department of Mathematics, University of Stockholm, Box 6701, S-113 85, Stockholm, SWEDEN

Department of Mathematics, University of Miami, Coral Gables, Florida 33124 